\title{
Community Perspectives on Research Consent Involving Vulnerable Children in Western Kenya
}

\author{
RACHEL VREeman \\ Indiana University (USA) \\ EUnice KamaARA \\ Moi University (Kenya)
}

Allan Kamanda

Moi Teaching and Referral Hospital (Kenya)

David Ayuku, Winstone Nyandiko, Lukoye Atwoli, Samuel Ayaya, and Peter Gisore

Moi University (Kenya)

Michael Scanlon and Paula Braitstein
Indiana University (USA)

\begin{abstract}
INVOLVING VULNERABLE PEDIATRIC pOpulations in international research requires culturally appropriate ethical protections. We sought to use mabaraza, traditional East African community assemblies, to understand how a community in western Kenya viewed participation of children in health research and informed consent and assent processes. Results from 108 participants revealed generally positive attitudes towards involving vulnerable children in research, largely because they assumed children would directly benefit. Consent from parents or guardians was understood as necessary for participation while gaining child assent was not. They felt other caregivers, community leaders, and even community assemblies could participate in the consent process. Community members believed research involving orphans and street children could benefit these vulnerable populations, but would require special processes for consent.
\end{abstract}

KEY WORDS: community-based research, pediatrics, sub-Saharan Africa, ethics, informed consent, Kenya

Received: July 15, 2012; revised: September 10, 2012

B IOMEDICAL AND BEHAVIORAL RESEARCH involving children presents complex ethical considerations. Children are classified as vulnerable subjects because they are considered unable to make autonomous decisions and must have their interests safeguarded. Kipnis (2003) identifies seven characteristics that may increase children's vulnerability in the research process: lacking the capacity to make rational decisions; being subjected to the authority of others; masking underlying dissent; undervalued or unrecognized rights; having acute medical conditions requiring immediate care; having serious medical conditions that cannot be effectively treated; and lacking access to needed social services. Among these vulnerable subjects, certain pediatric populations, such as orphaned or impoverished children, may be even more vulnerable.

Children's participation in research typically requires the consent of a parent or guardian. While parental consent is supposed to protect children, having one person consent on behalf of another also introduces additional complications to the informed consent process (Kodish, 2003). Parental informed consent may be influenced by multiple factors, including language and education barriers, differing views of health and research, and a desire to access health services for their children, particularly in disadvantaged settings (Eder, 2005). Ensuring an ethical informed consent process for the more vulnerable populations of children, such as orphans or children living in poverty, may thus be particularly difficult. A study of orphaned and HIV-infected children in Kenya suggested that parental consent procedures were inadequate in protecting children in research (Nyambedha, 2008). Determining what consent processes would best protect this particular pediatric population requires attention since the world has 132 million orphans and most live in resource-limited settings (UNICEF, Progress for children, 2010). Orphans bear a greater burden of poverty and ill health, and so the principle of justice would suggest that they should have access to research aimed at alleviating these burdens (UNICEF, Orphans, 2009). On the other hand, both their lack of one or both parents and the other factors associated with orphanhood-poverty, poor health, and other deprivations-increase their vulnerability and require additional protections. Thus, the ethical research involvement of particularly vulnerable populations of children merits further consideration.

In any setting, researchers must consider the role of the child in agreeing to research participation and

Journal of Empirical Research on Human Research Ethics, VoL. 7, No. 4, PP. 44-55. PRINT ISSN 1556-2646, ONLINE ISSN 1556-2654. @ 2012 By JOAN SIEBER. ALL RIGHTS RESERVED. PLEASE DIRECT ALL REQUESTS FOR PERMISSIONS TO PHOTOCOPY OR REPRODUCE ARTICLE CONTENT THROUGH THE UNIVERSITY OF CALIFORNIA PRESS'S RIGHTS AND PERMISSIONS WEBSITE, HTTP://WWW.UCPRESSJOURNALS.COM/REPRINTINFO.ASP. DOI: $10.1525 /$ jer.2012.7.4.44 
how the child's role evolves over the developmental and socio-cultural spectrum. Older children are typically involved in the decision-making process for informed consent through a process of pediatric assent (Baylis, Downie, \& Kenny, 1999). However, there are conflicting interpretations of pediatric assent (Nelson \& Reynolds, 2003; Wendler \& Shah, 2003), and the assent process depends on both a child's decision-making capacity and developmental trajectory (Kodish, 2005). Pediatric assent protocols vary widely, both among research institutions in the United States (Kimberly et al., 2006) and between countries (Vreeman, Nyandiko, \& Meslin, 2009). Furthermore, communities may have varying views on the autonomy and appropriateness of children to make these decisions (ibid.). For example, obtaining assent for children aged seven years and older is relatively common practice in some countries (Wendler, 2006); however, in a community-based study in Kenya, most participants were opposed to asking for assent in children younger than twelve or thirteen years of age (Molyneux et al., 2005). There is a paucity of research from resource-limited settings with regard to community perspectives on involving vulnerable children in research or how best to carry out processes such as informed consent for these populations (Mystakidou et al., 2009; Nyambedha, 2008).

As international research collaborations seek to learn how best to involve vulnerable pediatric populations in research, existing community structures for dialogue and decision-making may be helpful. In many Kenyan cultures, traditional community assemblies called mabaraza are used for both sharing information and gathering community opinions on issues (Naanyu et al., 2011). These mabaraza may be useful for both research investigation and development and the consent process, creating a culturally relevant venue for understanding community perspectives or beliefs. The mabaraza are typically larger than traditional focus groups, include a more heterogeneous population, and consist of more open discussion about the group's experiences (Naanyu et al., 2011). Traditional, formal community gatherings have been used with success to understand community perspectives on health programs in Kenya (ibid.) and on various aspects of biomedical research in Ghana (Tindana et al., 2011). In the context of a community in western Kenya that has hosted a long-standing partnership between a North American medical school and a Kenyan medical school (Einterz et al., 1995; Einterz et al., 2007; Inui et al., 2007), we sought to use traditional community assemblies (mabaraza) to understand the community perspective on the participation of vulner- able children in research. Our goal was to inform ethical research practices involving vulnerable children in this setting and in similar resource-limited settings, with a focus on the involvement of orphans and street children in research.

\section{Methods}

\section{Study Design Using Mabaraza}

We conducted a qualitative research study using dedicated mabaraza for the purpose of dialogic engagement with community members about pediatric research and informed consent in western Kenya. The mabaraza were officially organized by a Chief or Assistant Chief, who invited village elders and asked them to bring at least one caregiver of an orphaned or separated child, in addition to inviting their village community. We targeted recruitment of orphan caregivers because of our particular interest in the research involvement of vulnerable children, including orphans and street children, in this setting.

The mabaraza were employed much as focus group discussions are used for qualitative inquiry; they were conducted specifically for prompting group discussion and interactions about the community perspectives on particular content areas (Naanyu et al., 2011). Use of the baraza (singular form of mabaraza) was chosen specifically because it can yield more spontaneous and diverse individual and community perspectives than typical research focus groups (ibid.). We also wanted to capture the perspectives of community members in the specific county in which ongoing research work was underway. A trained facilitator and scripted question guide were used to guide the assemblies' discussion.

\section{Setting}

The study was conducted in the Uasin Gishu county of western Kenya under the auspices of the AMPATH Research Network. Uasin Gishu county is located in the Rift Valley province and constitutes three constituencies (Eldoret North, Eldoret East, and Eldoret South) (https://opendata.go.ke). Uasin Gishu county has a population of 894,179 people, of whom $38.6 \%$ live in an urban setting. Almost half of the population is estimated to live below the Kenya poverty line of 1,562 Kenyan shillings ( $\$ 18.75$ USD) per person per month in rural areas, and 2,913 Kenyan shillings (\$34.97 USD) per person per month in urban areas.

Kenya faces a huge burden from the HIV/AIDS epidemic. With a national HIV prevalence of $6.3 \%$, over 
1.3 million persons are estimated to be living with HIV (including 184,000 children) (Kenya Demographic and Health Survey 2008-09, 2010). In addition, over one million children in Kenya have been orphaned due to HIV (UNICEF, Info by country, 2009). Indiana University School of Medicine and Moi University School of Medicine have worked in a collaborative partnership for education, research, and clinical care in western Kenya since 1989 (Einterz et al., 1995). In 2001, Indiana University School of Medicine, Moi University School of Medicine, and Moi Teaching and Referral Hospital partnered to create AMPATH, an academic model for the provision of HIV care and other primary care services (Einterz et al., 2007; Inui et al., 2007). AMPATH has enrolled over 150,000 patients in western Kenya, and currently follows approximately 75,000 active patients (including over 24,500 children) at 69 healthcare facilities in western Kenya. AMPATH provides access to free antiretroviral treatment (ART), as well as comprehensive nutrition services, psychosocial support, and economic development training.

AMPATH has a highly functioning research network with shared North American and Kenyan leadership. AMPATH currently has over 110 IRB-approved research protocols underway. Ethical review is conducted by an Office of Human Resource Protectionsapproved institutional research and ethics committee.

\section{Study Population}

This study was conducted within the auspices of an ongoing longitudinal research evaluation intended to improve the health and well-being of orphaned children by evaluating the potential effects of their care environment on their physical health and psychosocial well-being (1R01HD060478-01A1). The parent study is using standardized site assessments, medical examinations, and psychosocial assessments to follow approximately 3,000 orphaned and separated children in the Uasin Gishu county of western Kenya for five years.

The goal of this study was to assess the community perspective on the participation of and consent for vulnerable children in biomedical and behavioral research within the Uasin Gishu county, where the parent study is taking place. For this purpose, two mabaraza were called in strategic peri-urban, densely populated locations within the county in which the parent study was taking place (Pioneer and Kapsoya locations). The mabaraza participants consisted of the provincial administration (the chief, assistant chief, and village elders), caregivers of orphaned and separated children, and other members of the general public, both male and female.

\section{Procedures}

The mabaraza were called by the community standards under the coordination of the chiefs and assistant chiefs. The assemblies were held in large, enclosed meeting rooms, one the municipal council hall and the other at the locational chiefs' camp. The mabaraza were conducted in June and July of 2011 in Kiswahili by a trained facilitator. Complete interview guides are available from the corresponding author upon request. Questions were based upon review of the literature, the input of local healthcare providers and researchers, and specialists in research ethics. The final questions covered multiple areas related to the experience of community-based research and consent and involving vulnerable children in research. The participants granted permission for audio recordings of the sessions to allow for later transcription. Field notes were taken during and immediately after the encounters. All of the recordings were transcribed and then translated into English by a trained translator. The study was approved by the Institutional Review Board of Indiana University in Indianapolis, Indiana, and by the Institutional Research Ethics Committee of Moi University School of Medicine in Eldoret, Kenya.

\section{Analyses}

A system of manual, progressive coding of the transcripts was used to identify emerging central concepts (Strauss \& Corbin, 1990). The initial stage of constant comparative analyses was done through open coding by two independent investigators (Vreeman and Kamaara), involving a line-by-line analysis of each transcribed page of informant data to elucidate meanings and processes. These independent analysts also extracted and compared themes. Before condensing the codes, the analysts read the data several times, including comparison of a final review of all open codes from each of the analysts, followed by re-coding based on consensus by three analysts (Vreeman, Kamaara, Scanlon). We then did axial coding, the process of relating categories to their subcategories and linking them together at the level of properties and dimensions (Strauss \& Corbin, 1990), to organize the themes into their causal relationships. Hypotheses and concepts were developed inductively from the data. Finally, relationships among the codes were integrated and refined. Selected quotations were used to illustrate key themes. 
We incorporated triangulation or verification on several levels. First, we analyzed and compared transcript data and field notes from mabaraza in two locations. Second, independent reading, coding, comparison, and summary of themes were performed by three investigators (Vreeman, Kamaara, Scanlon). Finally, we incorporated two sources of peer debriefing and peer checking of transcripts and themes (Braitstein, Kamanda).

\section{Results}

We collected data from 108 participants at the two mabaraza (79 male and 29 female). The Kapsoya baraza consisted of 37 participants, of which 17 were female and 20 male, including one chief, 11 village elders, and 25 caregivers or other community members. The Pioneer baraza consisted of 71 participants, 12 female and 59 male, including one assistant chief, 35 village elders, and 35 caregivers or community members.

\section{Community Knowledge and Attitudes Towards Research}

Community members generally understood research as a form of inquiry. As two community members from different locations shared: "establishing the cause of the problem is research" and "research is the search for the root cause of something." The mabaraza participants also expected that research would result in direct benefits for the community and that "those being involved in the research would get benefits." As one village elder described:

We will give consent so that the researcher can continue giving aid to the community. The community should benefit in different ways from the research.

While these benefits were sometimes characterized as information "useful for the overall growth of the community," some community members also expected concrete benefits such as new water sources, medicines, and tuition for children. Both the information gathered through the research and additional direct benefits were considered to "assist the community," and the indirect and direct benefits were at times conflated. For example, when asked about what the information was that would assist the community, a community member's first response was:

An example [of information] is something that can assist the community. For example, during the period of farming, the community can be given seed and fertilizer. In times of sickness, they can be given drugs.
Understanding the benefits of research was also considered a crucial component of the informed consent process. When asked about what information community members required in order to give informed consent, this participant's response summarizes their priorities: "Once we know the goal of the research, then next we need to know the benefits of the research."

\section{Participation of Vulnerable Children in Research}

In response to a general question asking "What do you think about having children participate in research?" participants widely endorsed the participation of vulnerable children in biomedical research. This endorsement seemed to spring directly from their belief that there would be individual benefits from research participation. One mother explained:

Some people carry out research and bring aid from abroad, but that aid doesn't reach the needful parties. The children should therefore be involved in research.

The mabaraza participants stressed that the more vulnerable children, particularly orphans and girls, should be able to participate in research and to reap these individual benefits as well. As two community members stated: "Involving the girl child in research is very important" and "Children should be involved in research, especially orphans." The community believed that both individual and community benefits would result from research participation, including protection, new knowledge to help in the care of children, improvement of their lives (through health improvement and through financial assistance), becoming more educated about health issues, and having HIV testing or other medical followup and care. Some thought that these benefits would extend to the child "being taken" by the research project, which meant that the research project would fully sponsor the child or take responsibility for the child's care and uptake.

Research was frequently viewed as an educational opportunity for impoverished children. Research participation could "create awareness of the future" and improve health literacy. As one participant summarized:

By involving the child in research, the child will be enlightened and ahead in many issues. They will be knowledgeable and therefore prepared for the future. If they are not involved in research and educated on different issues, they will not be knowledgeable. 
In addition to the belief that research would result in benefits to the participants, research was also closely equated to HIV diagnosis and care. Although the questions about research never involved HIV, the participants assumed that HIV testing would be a part of any research investigation. "When you involve a child in research, they will know if there is something good or bad for them in the future," said one participant, subsequently defining that "knowing" as the child knowing whether or not they had HIV. "Knowing the child's condition" because of research participation or "know[ing] the child inside out because of the research" (i.e., knowing "everything" about the child) was used to refer to knowing that the child had HIV. Learning the child's HIV status through research participation was viewed as a potential benefit, but also as a risk. For some, this was part of the reason to include children in research: "[Participating in research] is for the good of the girl child. They will be informed about HIV/ AIDS." For others, the consequences of HIV testing and diagnosis were something to be feared and a reason to avoid research participation, as expressed by a participant who said, "they will discover something bad.... and [the children] can get discouraged." Many participants believed that HIV disclosure to children might be a part of the research processes, expecting that the process of being informed during research consent might include being informed of one's HIV status, and they cautioned that "if you inform them about this issue, they will be shocked. You must know how you will handle them."

Despite the general belief that vulnerable children could benefit from research participation, the adults in the mabaraza also expressed some worries that they would have about research involving children. Many community members believed that the benefits of research would include significant care for the children's needs, and this led to concerns that the children might not be allowed to return home. "If you involve my child in research, will you adopt them or will you let them come back home?" asked one participant. Several highlighted a concern that research would lead to children participating in dangerous or bad behavior. For example, one participant expressed a worry that "if the child is misdirected by the research, they will deviate from the normal expectations and go into sexual immorality or theft." Another said, "Some types of research may ruin the child...stall their progress." Other worries about research participation centered around either the researchers or the children learning things that the parents did not want them to know. Examples of this sensitive information included HIV results, abuse, or pregnancy. For example, participants expressed the following fears about research involving children:
If the researcher discovers something negative about me through the child, as a parent, will I be at risk of imprisonment?

The school girl may be pregnant, and the parents are very harsh. When this girl participates or is involved in the research and is informed that she is pregnant, it will be devastating...the girl will be told about her pregnancy and will be sent home. The girl knows that she will neither go home, nor to school, so she may commit suicide by using drugs. That is one worry that will arise.

The participants also worried that children would share information that would "contradict what the parent may have answered." A few participants did express concern related to the experimental nature of research and harm resulting from research interventions. For example, one noted the concern that "when [the children] are researched on and something new is tried out on them, it could make them different from who they were." The participants felt that researchers could best prevent these negative things from happening by providing both the children and parents with information and education "so that they know good and bad." Follow-up of the child participants was also recommended; "researcher and parent or guardian should do a joint follow-up."

\section{Consent for Children to Participate in Research}

The mabaraza participants identified a child's parent or guardian as the appropriate person to give consent for children to participate in research, but a number of others were also considered acceptable givers of consent. These included teachers, older brothers, pastors, and, on occasion, the children themselves. Community consent for children's research participation was considered a potential substitute for parental consent in particular cases where the child is not receiving adequate care. Examples of these cases provided by the community included instances where a child was orphaned, the child was not receiving adequate care from the parent, or the child was "difficult to handle." As the participants explained, community consent could be sought as a substitute at "times when the child is not well taken care of" or "the community can be asked to step in when the parent dies and the child is orphaned." In these scenarios, the community was seen as a family substitute, both for care and for issues such as consent. "The community is a family," said one participant.

Whether and when children could consent for their own participation in research was a source of some disagreement and variation in responses, revealing 
heterogeneity within these community samples and differences in opinion from the national regulations. The groups reached some consensus that children could consent for their own participation when they reached a certain age or maturity level, and 18 years was the age generally given for when this could occur. It was also recognized that maturity would play a role, even at an age like 18 years; the child's ability to consent would "depend on the capability of the child," whether they could "grasp things easily" and even "if they have progressed out of their parents' home." Orphaned children with "no parent or guardian" and "street children [who] are under no one's rule and have no one to consent for them" were also brought up by some participants as children who could consent for themselves. Participants were not asked specifically about pediatric assent and dissent, but they did not bring up these forms of participation in consent or ways in which children would participate in the process if a parent was present.

\section{Orphaned Children Participating in Research}

The participants' beliefs about whether orphans should be involved in research revolved around orphans obtaining the perceived benefits of research. When questioned about potential concerns or worries about involving orphans in research, the participants focused on the risks of the orphans missing out on research benefits if they did not participate. In this light, the participants stressed that the orphans "should be treated equally, fairly, and be on the same level with the rest" of the family's children, and thus be allowed to participate in research. The participants expressed that "not all people have these children's best interests at heart" related to concerns that orphaned children would not be allowed research participation. They also felt that research involving orphans might benefit orphans more indirectly by helping the community identify ways to improve their care. The participants thought that, through research, the orphans and the community might be "taught, they should also be counseled and advised on how to live well."

The participants elaborated on the problems faced by orphans ("so many problems, including lack of proper care"), including their mistreatment by their caregivers, but they did not relate this discussion directly to research participation. Participants described general vulnerabilities faced by the orphans, including malnutrition, physical abuse, being denied access to school or care, and being used to solicit money. The participants continued to express beliefs that children in need might be "taken up" by the research team and provided with education, care, or even adoption as a result of research participation. For example, one suggested:

The children who are taken good care of should remain under that care [in the community], but those who have more problems or are mistreated should be taken up by the research team.

The participants did express concern that orphans taking part in research might "find out that they are sick [and] it will disturb them," referring to potential consequences of being diagnosed with HIV during research and having their status disclosed to them. They were concerned that research involvement might then result in "isolation" for the children because of stigma related to HIV.

While the participants had discussed community consent as an option for determining whether orphaned children could participate in research, they generally had a structured hierarchy of community members who they considered appropriate to consent for the child and who needed to be approached. The participants expected that orphaned children would not be completely on their own and would instead have a relative or some "key men or women that have been assisting" them. As one participant in the Kapsoya location described:

When a death occurs, it doesn't mean the whole family has died. Those children may be staying with other people from the family. Those who stay with the children should consent.

To determine who would be the appropriate person to consent, the participants primarily recommended "to go to the chief" or to "liaise with the government arm that deals with the children and get consent." The participants were able to mention specific government officials, such as the District Children's Officer, who would be responsible for guardianship of orphaned children in this setting. The participants recognized that some of the caregivers of orphaned children might be more or less likely to have the children's best interests at heart. For example, one participant stated: "An uncle will be less concerned for such a child.... It is the grandmother who will take good care."

The mabaraza participants felt that street children would require different consent processes. They described a number of reasons why they thought the children ended up living on the street, including having families who could not provide for them, the child being "too difficult" or "troublesome," or being unable to afford school enrollment. Both mabaraza felt that building personal relationships based on trust and "long-term friendship... [were needed to] achieve 
the objectives of your research." For children "who go back home" or do not spend all of their time on the street, the community members felt the children's parents should be approached for consent. The village elders were mentioned as people who should be able to inform researchers of the situation surrounding a street child. Many of the participants also suggest getting an authorization letter from the chief and being in contact with the District Children's Officer to facilitate legal consent. The "street children's leader" was also considered an important person to involve related to the child's participation in research. Although these various persons were mentioned as possible people to help give research consent for street children, the children were also considered by some to be able to consent for themselves:

For those whose parents are able and can be identified, the parents should give the consent, but for those who don't have parents, the children can decide for themselves.

However, many still stressed the importance of trying to involve the child's parents if at all possible:

Getting the child['s consent] will be up to you, but most important is that the parents consented, so they won't accuse you of taking advantage of them and involving the child in the research without their consent.

The participants felt that contacting the village elders, chiefs, or county personnel might be an initial process, followed by asking the children for consent directly so that "by the time we approach the street children, the legal requirements should have been fulfilled." Some participants expressed strong caution against trying to have the street children consent directly:

Legally, these children don't have the permission to consent for themselves because they are children, but because you want to involve them in research, they could consent and give you the information you require. You could go ahead and consent them, but that is illegal, and if you are found doing so, you will be arrested. You will have gone against the rule.

Community members also exhibited heterogeneity in their views on the appropriate age at which street children could consent for themselves. The ages for street children to be able to consent ranged from 6 years to 15 years of age, with stress being placed on the children's intelligence and knowledge. Some thought the street children would be able to consent even at very young ages:

Children aged six years and above are intelligent because they smoke bang and sleep on the streets. They know a lot. They know a lot and are alert.
Others thought the children would need to be into adolescence to be able to consent:

A six-year-old doesn't know much. Children aged fifteen years and above can make their decisions.

Children from age fifteen to seventeen are able to make their own decisions. A child below ten years cannot make a decision.

Community involvement was considered important to the participants in order for research with street children to go forward. "The community has to be asked or be involved. If the community accepts, then the researcher can go ahead," explained a participant in the Kapsoya group. Community consent was considered a possibility if it seemed clear that the research was going to benefit the child: "That's one reason for the community to consent-because the research will benefit these children."

\section{Discussion}

In community gatherings, chiefs, village elders, and caregivers of children in western Kenya described their beliefs about having children participate in research. The mabaraza participants generally viewed research participation as having the potential to benefit children directly, and they strongly endorsed even the participation of more vulnerable groups of children. When describing the risks to research, they focused on a perceived risk to missing out on the presumed benefits of research. Participants did identify risks to research participation such as learning about sensitive information or experimental interventions resulting in harm. The community endorsed parental informed consent for children's research participation, but they also supported having other caregivers, community leaders, and even community assemblies to participate in the consent process. Research involving orphans and street children was considered to have benefits for these vulnerable populations, but to require special processes for consent.

Informed consent involving children was generally conceptualized as a process that involved the adult responsible for the child and the researcher, but not the child. Requiring the children to give assent or agreement to participate in research was never raised by any of the baraza participants, nor was the idea that a child's dissent could override parental consent. The parents or caregivers recognized that a child might give answers that contradicted their own in response to research questions, but the child disagreeing on research participation was not raised as a consideration. Since the groups were not 
probed specifically about assent or dissent, we cannot conclude whether these forms of participation were not prominent in their minds or whether they had substantive disagreement with them. Directed questions did assess their views on children's participation in the consent process for orphans and street children, for whom obtaining parental informed consent might be difficult or impossible. The absence of consideration of assent fits with the reaction of community members in another part of Kenya, who "reacted with surprise" when asked about having children assent and had "general agreement that children should not be asked" (Molyneux et al., 2005).

Some of the community members participating in these mabaraza did see a role for children to provide their own consent if they were orphans over the age of 18 years or if they were older street children. The community's perspectives are consistent with the legal age of majority, which is 18 years in Kenya, but they do not necessarily fit with the guidelines currently in place for informed consent processes involving children in this setting. In Kenya, the guidelines for the "Ethical Conduct of Biomedical Research Involving Human Subjects" published by the Kenya National Council for Science and Technology do not support a specific age or role for pediatric assent, but they do support respecting the dissent of pediatric research subjects, stating that if a "child refuses to participate in the research, that refusal must be respected unless there is no other medical alternative from which the child could benefit" (National Council for Science and Technology, 2004). The possibility of child dissent was never mentioned by any of the study participants, and their discussions seemed to assume that most children would comply with their parents' or guardians' wishes.

For biomedical and behavioral researchers considering implementation of an ethical informed consent process in Kenya, these findings suggest that it is important to understand that informed consent in this setting may be complicated by difficulties in distinguishing research from health interventions or in adjusting the expectations of assistance. These findings are somewhat congruent with research done on the coast of Kenya that suggested that consent was not based on the details of the research protocol (Molyneux et al., 2005). In our work, the participants expressed that the potential benefits of the research would be scrutinized-rather than the protocol. In the study from the coast, consent was based more on broad trust in the institution involved (ibid.). Trust was not a prominent theme within these mabaraza, but participants did want to understand the intent of the proposed research and to ensure that the intent was for the benefit of the community and the children involved. In these peri-urban, slum locations, community members had strong expectations of children receiving aid in exchange for research participation, which may be a particular concern in settings with the vulnerable combination of lower research literacy, poorer access to healthcare and other services, and limited financial resources.

The idea that children might "be taken" or have their physical and educational needs met in a way that might lead to adoption has previously been highlighted as a concern for social science research as well (Nyambedha, 2008). These findings have implications for the detail and clarity needed in the informed consent processes for this population and how important it will be for researchers to ensure that the procedures, risks, and benefits to their proposed work are all well understood. As even well-intentioned caregivers in similar resource-limited settings may be thinking primarily of the perceived benefits of the study, local research ethics committees and investigators alike need to pay special attention to whatever precautions may be needed to safeguard children's best interests in that particular context. Involving the community in review of research proposals in assemblies such as mabaraza or engaging community leaders tasked with the protection of children were widely perceived as culturally acceptable and important protective measures to further this goal.

The community participants in these settings made a strong connection between research and activities related to HIV testing, treatment, and disclosure. This connection shaped how they viewed the goal, risks, and activities of research. In reality, HIV treatment is available at no cost to HIV-infected individuals, with no requirement for research participation. While this research project did not focus on HIV and was not described as an activity of the AMPATH partnership in western Kenya, the prominence of this HIV care program may have resulted in some of this effect. AMPATH has conducted both research and community-based testing within these locations, and multinational research teams may be assumed to be conducting HIV-related activities for this reason. Researchers working in other settings where HIV care is one of the few accessible healthcare systems, where HIV-related stigma is a prominent community concern, or where HIV-related research is the primary type of research with which a community may have experience, may find similar assumptions within the community. These beliefs need to be taken into account throughout the research implementation process. 
The nature of this study results in some limitations that merit consideration. This qualitative inquiry relies on contextual data and the shared experiences of subjects in two locations in western Kenya-a very particular part of the world. These contextual data may not be generalizable to other geographic locations or other cultures, even within the same country. In addition, this methodology draws data from a relatively small convenience sample, which can also limit generalizability. Community data gathered from these resource-limited slum locations provide an excellent picture of their understanding of research, but it may not be applicable to more rural areas or areas with less economic deprivation. In addition, these qualitative data reflect a rich diversity of opinions even within this particular group; community members' beliefs and perceptions varied, belying beliefs that one could easily categorize the culture of a resource-limited setting. Nonetheless, these data illuminate the perception of pediatric research in this population, and the groups' responses had good thematic saturation. Moreover, the attitudes and beliefs of participants in this particular resource-limited setting may be more applicable to the attitudes and beliefs of participants in other resource-limited settings than the existing data from resource-rich settings.

To our knowledge, there have been no other published findings from mabaraza or traditional assemblies around the issue of pediatric research participation, so this particular sample provides unique contextual data for ethical pediatric research practices. The baraza form itself has some limitations in that it does not lend itself to getting responses from every participant to every question because of the large size of the gathering. While there may be less depth to the response to a particular question, it does provide a community overview, with a focus on the community leaders, and broader perspective on the community beliefs than more intensive individual interviews or focus groups are likely to provide. It is possible that the presence of community leaders may have made some of the participants less likely to speak, with particular concern for not hearing the voices of women, younger adults, the poor, or anyone else more marginalized in this society. In an attempt to counter this, at various points, the facilitator specifically asked for the women and for those who had not yet spoken to offer answers. Overall, in this context, the mabaraza were useful not only for gathering this community perspective, but also for trialing the use of this structure for further endeavors in community engagement or community consent for research.

The data collected in these mabaraza provide insight into how the community in western Kenya views children, their participation in research, and appropriate consent processes for medical and behavioral research. Community members may interpret the benefits and risks of research participation in strikingly different ways than the researchers, and their understanding must therefore be evaluated and enhanced by ongoing dialogue about what it means for children to participate in a research project. Reconciling differences among community preferences, national guidelines, and international guidelines remains a challenge, and yet the community assemblies offer a venue in which to discuss these issues. The data from these community assemblies fill gaps in the current research on the ethics of involving children in research (Vreeman et al., 2009), offering insight into the understanding of pediatric consent in resource-limited settings and how pediatric involvement might be discussed and regulated by the community.

\section{Best Practices}

Biomedical and behavioral researchers in resource-limited settings should consider the extent to which communities may expect benefits to children or to the community for research participation. In settings where communities have limited access to healthcare, researchers need to be particularly cognizant of the potential for research to be associated with direct health interventions. Assessing the community's understanding of children's rights and decision-making capacity and adapting research processes for consenting caregivers and assenting children are necessary initial steps in multinational research involving children. Researchers should attempt to engage with communities in an informative consent process that includes careful discussion of procedures, risks, and benefits. Moreover, researchers must work with the community to determine acceptable practices for consent involving pediatric populations at particular risk, including orphans and street children. Traditional community assemblies, such as mabaraza in Kenya, may offer an appropriate venue in which to examine the particular issues for involving children in research and through which to reach consensus with the community about appropriate research practices.

\section{Research Agenda}

This study highlights several areas where additional data are needed to guide the participation of children in biomedical and behavioral research in resource-limited settings. For a particular setting or cultural context, researchers should elicit the community perspective on pediatric research participation. Understanding how a given population perceives research, consent processes, 
and the benefits and risks to children can guide future research endeavors. Second, research is needed on the ongoing participation of communities throughout the course of a multinational research project. Particularly in resource-limited settings, researchers need to explore how to best communicate with the community before research is commenced, how to involve the community during research, how to disseminate findings after research completion, and how these interactions impact the research and outcomes. Further research is also needed to define the best practices for engaging in pediatric consent and assent in a context like Kenya. Investigating the face validity and reliability of tools to elicit caregiver consent and pediatric assent and evaluating the age-specific comprehension of the materials used in the course of consent and the research process remain important items for the research agenda. Lastly, while mabaraza in Kenya may represent an important venue to better understand appropriate consent and assent processes as well as community perspectives on children in biomedical and behavior research, further data are needed on the use of these types of community assemblies in research settings.

\section{Educational Implications}

This exploration of the community perspective on children's participation in research in western Kenya has a number of educational implications. First, both investigators and members of the involved research ethics committees would benefit from training in the culturally and community specific view of pediatric research participation. In addition to training in the ethics of pediatric research, understanding the community perspective would assist in developing consent and assent processes that best protect this vulnerable population. Second, because of the community's view on research risks and benefits and because of the limited experience with implementing pediatric assent within this setting, study personnel in research involving children need to be trained on conducting a dynamic process of caregiver consent and pediatric assent. Study personnel will need to be trained on using open-ended questions and probes in order to evaluate families' understanding, fears, and relational dynamics. Finally, this compiled evidence supports the need to champion increased community involvement in research processes that include children. With meaningful community involvement, international researchers will best be able to develop an authentic perception of the community's views on children in research and how this vulnerable population can be responsibly involved in biomedical and behavioral research.

\section{Acknowledgments}

We would like to acknowledge the work of Eunice Walumbe in the transcription and translation of the mabaraza proceedings. This work was funded by a grant from the National Institute of Child Health and Human Development (NICHD) to Dr. Paula Braitstein (1R01HD060478-01A1) to improve the health and well-being of orphaned and separated children. Dr. Vreeman was also supported by a grant from the National Institute for Mental Health (NIMH) (1K23MH087225-01.) In addition, this research was supported by a grant to the USAID-AMPATH partnership from the United States Agency for International Development as part of the President's Emergency Plan for AIDS Relief (PEPFAR).

\section{Author Note}

Address correspondence to: Rachel C. Vreeman, Children's Health Services Research, Health Information and Translational Sciences Building, Suite \#1000, 410 W. 10th St., Indianapolis, IN 46202. Phone: 317-278-0552; Fax: 317-278-0456; E-MAIL: rvreeman@iupui.edu.

\section{Authors' Biographical Sketches}

Rachel Vreeman is Assistant Professor of Pediatrics at Indiana University School of Medicine and Co-Director for Pediatric Research for the Indiana UniversityKenya Partnership and Academic Model Providing Access to Healthcare (AMPATH). She has extensive research experience in western Kenya that focuses on improving the provision of healthcare to children in resource-limited settings, with particular expertise in children's adherence to HIV treatment. She led the development, analysis, and writing of this manuscript.

Eunice Kamaara is Professor in the Department of Philosophy and Religious Studies at Moi University in Eldoret, Kenya. She is completing a Masters in International Health Research Ethics, with a focus on informed consent in postcolonial contexts. She assisted in the analysis, writing, and revision of the manuscript.

Allan Kamanda is the study coordinator for the Orphaned and Separated Children's Assessments Related to their Health and Well-being (OSCAR) research study, which follows the outcomes of several thousand orphaned and separated children in western Kenya. He participated in the development of this project, facilitated each of the baraza assemblies, and participated in the writing and revision of this manuscript.

David Ayuku is Associate Professor in the Department of Behavioural Sciences, School of Medicine, Moi University 
College of Health Sciences in Eldoret, Kenya, and Co-Principal Investigator for the OSCAR research study in western Kenya. His research interests focus on the issues of street children and orphans. He participated in the development of this project and in the revision of this manuscript.

Winstone Nyandiko is Associate Professor and the Head of the Department of Child Health and Paediatrics, School of Medicine, Moi University College of Health Sciences in Eldoret, Kenya, and Associate Program Manager for the AMPATH partnership. His expertise lies in evaluating the clinical management of HIV-exposed and HIV-infected children and implementation research in Kenya. He contributed to the development of this analysis and the writing and revision of this manuscript.

Lukoye Atwoli is Lecturer in the Department of Mental Health in the School of Medicine, Moi University College of Health Sciences in Eldoret, Kenya, and Secretary of the Kenya Psychiatric Association. His research interests are psychotrauma in children and adults, general hospital psychiatry, and the interface between HIV and mental health. He contributed to the development of this analysis and manuscript.

Samuel Ayaya is Professor in the Department of Child Health and Paediatrics, School of Medicine, Moi University College of Health Sciences in Eldoret, Kenya, and Co-Chair of the Kenya Pediatric Research Working Group. His research interests include children's nutrition, growth, and development, the progression of HIV in children, and child abuse and neglect. He contributed to the project and manuscript development.

Peter Gisore is Lecturer in the Department of Child Health and Paediatrics, School of Medicine, Moi University College of Health Sciences in Eldoret, Kenya. His interests center around clinic- and community-based research on maternal and newborn health. He participated in the development of this project and this manuscript.

Michael Scanlon is Pediatric Global Clinical Research Scholar with Indiana University School of Medicine and the AMPATH partnership. He is focused on research to support long-term care of children with HIV in resourcelimited settings. He participated in the analysis and the development and revision of the manuscript.

Paula Braitstein is Associate Research Professor of Medicine at Indiana University School of Medicine, Co-Field Director for Research for AMPATH, and Co-Principal Investigator for the OSCAR research study in western Kenya. Her research expertise lies in evaluating care outcomes including for HIV-infected patients, orphans, separated children, and other vulnerable populations. She led the development of this project and contributed significantly to the writing of the manuscript.

\section{References}

Baylis, F., Downie, J., \& Kenny, N. (1999). Children and decisionmaking in health research. IRB, 21(4), 5-10.

EDER, M. (2005). Testing drugs in developing countries: Pediatric research ethics in an international context. In E. Kodish (Ed.), Ethics and research with children: A casebased approach (pp. 241-261). New York: Oxford University Press.

Einterz, R. M., Kelley, C. R., Mamlin, J. J., \& Van Reken, D. E. (1995). Partnerships in international health: The Indiana University-Moi University experience. Infectious Disease Clinics of North America, 9(2), 453-455.

Einterz, R. M., Kimaiyo, S., Mengech, H. N., KhwaOtsyula, B. O., Esamai, F., Quigley, F., \& Mamlin, J. J. (2007). Responding to the HIV pandemic: The power of an academic medical partnership. Academic Medicine, 82(8), 812-818.

Inui, T. S., Nyandiko, W. M., Kimaiyo, S. N., Frankel, R. M., Muriuki, T., Mamlin, J. J., Einterz, R. M., \& SIDLE, J. E. (2007). AMPATH: Living proof that no one has to die from HIV. Journal of General Internal Medicine, 22(12), 1745-1750.
Kenya Demographic and Health Survey 2008-09. (2010). Calverton, MD: Kenya National Bureau of Statistics and ICF Macro.

Kimberly, M. B., Hoehn, K. S., Feudtner, C., Nelson, R. M., \& SCHreIner, M. (2006). Variation in standards of research compensation and child assent practices: A comparison of 69 institutional review board-approved informed permission and assent forms for 3 multicenter pediatric clinical trials. Pediatrics, 117(5), 1706-1711.

KIPNIS, K. (2003). Seven vulnerabilities in the pediatric research subject. Theoretical Medicine and Bioethics, 24(2), 107-120.

Kodish, E. (2003). Informed consent for pediatric research: Is it really possible? Journal of Pediatrics, 142(2), 89-90.

Kodish, E. (2005). Ethics and research with children: An introduction. In E. Kodish (Ed.), Ethics and research with children: A casebased approach (pp. 3-25). New York: Oxford University Press.

Molyneux, C. S., Wassenaar, D. R., Peshu, N., \& Marsh, K. (2005). "Even if they ask you to stand by a tree all day, you will have to do it (laughter)...!": Community voices on the notion and practice of informed consent for biomedical research in developing countries. Social Science and Medicine, 61(2), 443-454. 
Mystakidou, K., Panagiotou, I., Katsaragakis, S., Tsilika, E., \& PARPA, E. (2009). Ethical and practical challenges in implementing informed consent in HIV/AIDS clinical trials in developing or resource-limited countries. SAHARA-J: Journal of Social Aspects of HIV/AIDS, 6(2), 46-57.

Naanyu, V., Sidle, J. E., Frankel, R. M., Ayuku, D., NyANDIKo, W. M., \& INUI, T. S. (2011). Rooting inquiry in tradition: The health baraza as a tool for social research in Kenya. Qualitative Health Research, 21(1), 14-26.

National Council For Science And Technology (NCST). (2004). Guidelines for ethical conduct of biomedical research involving human subjects in Kenya. Nairobi: NCST.

Nelson, R. M. \& Reynolds, W. W. (2003). We should reject passive resignation in favor of requiring the assent of younger children for participation in nonbeneficial research. American Journal of Bioethics, 3(4), 11-13.

Nyambedha, E. O. (2008). Ethical dilemmas of social science research on AIDS and orphanhood in Western Kenya. Social Science and Medicine, 67(5), 771-779.

Strauss, A. \& Corbin, J. (1990). Basics of qualitative research: Grounded theory procedures and techniques. Newbury Park, CA: Sage Publications.
Tindana, P. O., Rozmovits, L., Boulanger, R. F., Bandewar, S. V., Aborigo, R. A. ET AL. (2011). Aligning community engagement with traditional authority structures in global health research: A case study from northern Ghana. American Journal of Public Health, 101(10), 1857-1867.

UNICEF (United Nations Children's Fund). (2010). Progress for children: Achieving the MDGs with equity (vol. 9). New York: UNICEF.

UNICEF. (2009). Orphans. Retrieved from http://www.unicef. org/media/media_45279.html.

UNICEF. (2009). Info by country. Retrieved from http://www. unicef.org/infobycountry/kenya_statistics.html.

Vreeman, R. C., Nyandiko, W. M., \& Meslin, E. M. (2009). Pediatric assent for a study of antiretroviral therapy dosing for children in Western Kenya: A case study in international research collaboration. Journal of Empirical Research on Human Research Ethics, 4(1), 3-16.

Wendler, D. S. (2006). Assent in paediatric research: Theoretical and practical considerations. Journal of Medical Ethics, 32(4), 229-234.

Wendler, D. \& Shah, S. (2003). Should children decide whether they are enrolled in nonbeneficial research? American Journal of Bioethics, 3(4), 1-7. 
Reproduced with permission of the copyright owner. Further reproduction prohibited without permission. 